\title{
The intercellular adhesion molecule, cadherin-10, is a marker for human prostate luminal epithelial cells that is not expressed in prostate cancer
}

\author{
Marjorie M Walker ${ }^{1}$, Sebastien M Ellis², Michael J Auza ${ }^{2, *}$, Anup Patel ${ }^{3}$ and Peter Clark ${ }^{2}$ \\ ${ }^{1}$ Division of Investigative Sciences, Department of Histopathology, St Mary's Campus, Imperial College \\ London, London, UK; ${ }^{2}$ Leukocyte Biology Section, National Heart and Lung Institute, South Kensington \\ Campus, London, UK and ${ }^{3}$ Department of Urology, St Mary's NHS Trust, Paddington, London, UK
}

\begin{abstract}
During the normal turnover of prostate epithelium, stem cells in the basal cell layer produce an intermediate cell population that gives rise to fully differentiated secretory luminal cells. This process is extensively studied in relation to the development of prostate disease, in particular, to elucidate the origin and nature of prostate cancer. We previously showed that the mRNA of a poorly characterised intercellular adhesion molecule, cadherin-10, is strongly expressed in human prostate. Using anticadherin-10 antibodies, immunohistochemistry, and confocal microscopy, we have examined the pattern of cadherin-10 expression in relation to human prostate epithelial differentiation markers (E-cadherin, CD44, and cytokeratins (CK) 14, 18 and 19) in archival paraffin-embedded and fixed-frozen histopathological specimens in individual and serial sections. In nonneoplastic prostate, E-cadherin is expressed by all basal and luminal epithelial cells, while cadherin-10 is variably expressed in luminal cells where it is colocalised with E-cadherin at basolateral plasma membranes. Cadherin-10 is absent in CK14- and/or CD44-positive basal cells, but is expressed in CK18-positive luminal cells (differentiated secretory cells), a subset of CK19-positive intermediate/luminal cells, but not CK19-positive basal cells. Small foci of prostate cancer express E-cadherin, CK19 and CK18, but cadherin-10 expression is low or undetectable. These findings suggest that the expression of cadherin-10 is associated with the later stages of differentiation of luminal secretory cells, indicating a specific role in secretory cell terminal differentiation. While prostate cancer cells express secretory cell markers (eg, CK18, prostate-specific antigen) and the more generally expressed E-cadherin, their failure to express cadherin-10 further emphasises a role for this cadherin in normal prostate organisation and function.
\end{abstract}

Modern Pathology (2008) 21, 85-95; doi:10.1038/modpathol.3800988; published online 14 December 2007

Keywords: cadherin; cancer; CD44; confocal microscopy; cytokeratin; immunohistochemistry

In human prostate, the glandular epithelium consists of a layer of terminally differentiated cuboidal/ columnar luminal secretory cells overlying basal cells. ${ }^{1}$ The basal cell layer is believed to contain a subpopulation of stem cells that produce transit amplifying cells whose progeny undergo a programme of differentiation, via intermediate

Correspondence: Dr P Clark, PhD, Leukocyte Biology Section, National Heart and Lung Institute, Sir Alexander Fleming Building, South Kensington Campus, Imperial College London, London, UK.

E-mail: p.clark@imperial.ac.uk

*Current address: School of Medicine, University of Virginia, Charlottesville, Virginia 22908, USA.

Received 23 July 2007; revised 21 September 2007; accepted 01

October 2007; published online 14 December 2007 cells, towards fully differentiated luminal secretory cells. $^{2-7}$ In human prostate tissue samples, the glandular epithelium typically exhibits phenotypic heterogeneity reflecting stages of differentiation during turnover and repair. ${ }^{5}$ Understanding this differentiation process is crucial to a more complete understanding of the origin and development of prostate disease, in particular, prostate cancer.

Prostate cancer cells express a number of components that are markers of secretory luminal cells (eg, cytokeratin (CK) 8 and 18, prostate-specific antigen). ${ }^{2-6}$ This was initially interpreted as suggesting that they arose from transformed luminal epithelium, however, it is now known that both androgen-dependent and androgen-independent 
prostate cancer cells also express basal and intermediate cell characteristics that are normally lost by differentiating luminal cells (eg, CK19). Such evidence indicates that prostate cancer arises from malignant transformation of intermediate cells, and that the expression of luminal cell markers in these cancer cells is due to the aberrant differentiation of transformed intermediate cells. ${ }^{2-6}$

Intercellular adhesion is a key factor in epithelial tissue morphogenesis and maintenance, and perturbation of this adhesion is of considerable importance in cancer. Cadherins are a family of $\mathrm{Ca}^{2+}$-dependent cell-cell adhesion molecules with well-established roles in cell-cell recognition, intercellular junction organisation and cell differentiation. $^{8,9}$ The role of cadherins, particularly the prototypical classical cadherin, epithelial (E)-cadherin, has been studied in detail in relation to metastatic potential and prognosis in carcinoma. ${ }^{10-13}$ In general, loss of E-cadherin expression or function is associated with more aggressive tumours and poor prognosis. Expression of E-cadherin is typically reduced in prostate cancer. ${ }^{14-19}$ Some studies have found an association between E-cadherin expression and prostate cancer grade or stage, while others have failed to do so; however, loss of E-cadherin and the onset of expression of N-cadherin are observed in high-grade prostate cancer. ${ }^{17,20-22}$ We previously found strong expression of cadherin-10 mRNA in human prostate. ${ }^{23}$ The mRNA of this molecule is most strongly expressed in the central nervous system, ${ }^{24}$ and we raised antiserum to show that the protein is present in blood-brain barrier capillaries, and in some ependymal epithelia, but not in nonbrain vessels. ${ }^{23}$ Here, we use immunohistochemistry and confocal microscopy to examine the expression and localisation of cadherin-10 protein, in relation to known differentiation markers, in non-neoplastic and neoplastic human prostate tissue. The pattern of expression that we find suggests a crucial function for cadherin-10 in prostate epithelial differentiation and pathogenesis.

\section{Materials and methods}

\section{Human Tissues}

Prostate tissue samples were from patients (at St Mary's NHS Trust, London) undergoing either needle biopsies or radical prostatectomies for biopsy-proven adenocarcinoma. All tissues were obtained with local ethics committee approval and informed consent. Immunostaining for cadherin 10 was performed in seven cases of benign prostate adenomyomatous hyperplasia, mean age 71 years, (range, 54-84) and seven cases of prostate adenocarcinoma, mean age 65 years (range, 63-69). Three cases showed concurrent HGPIN in the sections examined. Gleason score and stage are shown in Table 1.
Table 1 The Gleason scores and staging of patient samples exhibiting prostate adenocarcinoma

\begin{tabular}{lcc}
\hline Age (years) & Gleason score & Stage \\
\hline 63 & $4+3,7$ & PT3c \\
67 & $3+3,6$ & PT2c \\
69 & $3+2,5$ & PT2c \\
63 & $4+3,7$ & PT3a \\
62 & $3+4,7$ & PT2b \\
64 & $3+4,7$ & PT2c \\
65 & $3+4,7$ & PT3a
\end{tabular}

\section{Immunohistochemistry}

Paraffin wax-embedded prostate material was obtained from archival patient samples and fresh material from surgical resections, fixed in $4 \%$ paraformaldehyde in phosphate-buffered saline (PBS) and processed using standard protocols. Immunohistochemical staining was carried out as described previously. ${ }^{23}$ Briefly, $5 \mu \mathrm{m}$-thick sections were de-waxed and hydrated. Endogenous peroxidases were deactivated by incubation in hydrogen peroxide solution, and then sections were rinsed and blocked in $5 \%$ normal goat serum $/ 5 \%$ human ABO serum in PBS for $30 \mathrm{~min}$. Affinity-purified rabbit anticadherin-10 antibody at a concentration of $3 \mu \mathrm{g} / \mathrm{ml}$ in blocking solution was applied to sections on slides and incubated overnight in a humidified chamber at $4^{\circ} \mathrm{C}$. Mouse monoclonal antibodies to various differentiation markers were individually applied to separate sections at the concentrations indicated below. Control rabbit or mouse IgG (Vector Laboratories, UK), in place of primary antibody, was used as a control for non-specific staining. Sections were rinsed in blocking solution three times for $10 \mathrm{~min}$ each, and horseradish peroxidase-conjugated goat anti-rabbit (or anti-mouse) antibody (Santa Cruz Biotechnology Inc., USA) $(3 \mu \mathrm{g} / \mathrm{ml}$ in blocking solution) was applied to the sections, incubated for $2 \mathrm{~h}$ in a humidified chamber at room temperature and rinsed. Peroxidase activity was detected using a DAB substrate development kit (Vector Laboratories, UK) and colour development was monitored. Some sections were counterstained with haematoxylin, dehydrated and mounted with coverslips using DPX mounting medium. The sections were examined and images digitally captured, using a Nikon Eclipse (E400) microscope (Tokyo, Japan) fitted with a Nikon Digital DXM1200 camera using Lucia imaging software (Prague, Czech Republic).

\section{Immunofluorescence and Confocal Microscopy}

Fresh material from surgical resection was immersion-fixed with the minimum possible delay in $4 \%$ paraformaldehyde in PBS overnight at $4^{\circ} \mathrm{C}$. The tissue was briefly rinsed in PBS then immersed in cryoprotectant, $10 \%(\mathrm{w} / \mathrm{v})$ sucrose in PBS, for 
$24 \mathrm{~h}$ at $4^{\circ} \mathrm{C}$. Cryoprotected tissues were placed in moulds with OCT embedding compound (VWR International, UK) and frozen in liquid nitrogen. Cryosections $(5-10 \mu \mathrm{m})$ were immunostained as described previously. ${ }^{23}$ Briefly, sections were brought to room temperature, permeabilised in $0.5 \%$ Triton X-100 in PBS for $30 \mathrm{~min}$ and blocked in $5 \%$ normal goat serum $/ 5 \%$ human $\mathrm{ABO}$ serum in PBS for $1 \mathrm{~h}$. Primary antibodies were applied in pairs of rabbit anticadherin-10 antibody at $1 \mu \mathrm{g} / \mathrm{ml}$ in blocking solution, together with one of a panel of mouse monoclonal antibodies to differentiation markers: anti-human CK14 (clone LL002) $1 \mu \mathrm{g} / \mathrm{ml}$ (Chemicon, Germany); anti-CD44 (clone F10-44-2) $1 \mu \mathrm{g} / \mathrm{ml}$ (Serotec Ltd, UK); anti-CK19 (clone MAB3238) $10 \mu \mathrm{g} / \mathrm{ml}$ (Chemicon, Germany); antiCK18 (clone CY-90) 1:1000 dilution of ascites fluid (Sigma, UK); anti-E-cadherin (clone HECD, a gift from Dr Vania Braga, Imperial College London 1:1000 dilution of hybridoma culture medium). As controls for non-specific staining, a parallel set of sections was incubated with a mixture of equivalent concentrations of control rabbit and mouse IgGs (Vector Laboratories, UK) in place of the primary antibodies. Sections were incubated with primary antibody mixes overnight at $4^{\circ} \mathrm{C}$, washed as above, then incubated with a mix of fluorescently conjugated secondary antibodies (Alexa 488 goat anti mouse IgG and Alexa 568 goat anti rabbit IgG (Invitrogen, UK) each $1 \mu \mathrm{g} / \mathrm{ml}$ ) at room temperature for $2 \mathrm{~h}$. Some sections were also stained with the nucleic acid stain, Topro 3 (Invitrogen, UK), as a nuclear counterstain. After washing, sections were mounted with coverslips using Vectashield Hard-Set mounting medium (Vector Laboratories, UK) and examined using a Leica TCS-NT laser scanning confocal microscope (Leica Microsystems, Germany) fitted with a Krypton/Argon laser. Single confocal sections from the mid-level of the tissue sections were digitally captured using the confocal system software. Where comparisons of staining levels were made, confocal settings were kept constant.

\section{Results}

\section{Cadherin-10 Expression in Normal Human Prostate} Epithelium is Heterogeneous

In morphologically normal gland profiles, the expression of cadherin-10 in prostate epithelial cells was seen to vary both between and within individual glandular profiles (Figure 1). Many glandular profiles were strongly positive for cadherin-10 immunoreactivity, while others exhibited weak, or no, staining. In some profiles, it was apparent that cadherin-10 expression levels could abruptly change between high and low expression (Figure 1c-e). Immunohistochemical and immunofluorescence localisation of cadherin-10 indicated that this molecule was expressed at the basolateral membranes of luminal cells, and was absent from basal cells, though strong staining at the basal membranes of luminal cells could indicate expression in basal cells at regions of contact with luminal cells (Figure 1d). Confocal microscopy after costaining of cadherin-10 and E-cadherin in fixed/frozen sections showed that when they were coexpressed in the luminal epithelium, they occupied the same basolateral membranes (Figure 1ei-iii).

\section{Cadherin-10 and Basal Cell Markers in Normal Human Prostate Epithelium}

CD44 and CK14 are well-established markers of prostate basal cells. However, it is known that some alternatively spliced variants of CD44 are expressed by luminally located intermediate cells, ${ }^{25}$ and these isoforms would be detected by pan-CD44 antibodies, such as used in this study. In addition, CK14 expression in basal cells is lost earlier than other basal cell markers, as cells undergo differentiation towards intermediate cells, ${ }^{6}$ so that this marker will not be detected in all cells in basal positions.

Using immunohistochemical localisation in morphologically normal glands, anti-CD44 strongly labelled basal epithelial cells with little obvious staining of luminal cells (Figure 2a). Immunofluorescence and confocal microscopy, using the same antibody, revealed that while CD44 was strongly expressed in basal cells (Figures 2b, c and di-iii), it was also variably present in the basolateral plasma membranes of a subset of luminal cells (Figure 2dii). In glands where CD44 and cadherin-10 are both present in luminal cells, cadherin-10 is generally more strongly expressed in weakly CD44-positive cells (Figure 2di-iii); although not all CD44 weakly positive cells strongly express cadherin-10.

Although CK14 was observed in the basal cells in many normal glands (Figure 3), it is absent from others (Figure 3diii). Double staining of CK14 and cadherin-10 emphasised their distinct distribution: colocalisation was not observed, CK14 being confined to basal cells and cadherin-10, when present and luminal cells (Figure $3 \mathrm{~b}-\mathrm{d}$ ).

\section{Cadherin-10 and the Intermediate Cell Marker, CK19, in Normal Human Prostate Epithelium}

In morphologically normal prostate epithelium, CK19 is considered to be a specific intermediate cell marker. ${ }^{3,7,26-28}$ In some glandular profiles, CK19 was strongly expressed in basal cells, but weak or absent in luminal cells, while in other glands, cells in luminal positions strongly expressed CK19 (Figure 4). CK19-positive cells can appear to be intercalated between CK19-negative luminal cells in some glands (Figure 4). Cadherin-10 is not present in CK19 positive cells in basal positions (early intermediate cells) (Figure 4b, $\mathrm{c}$ and di-iii). CK19 was strongly expressed in a subset of 

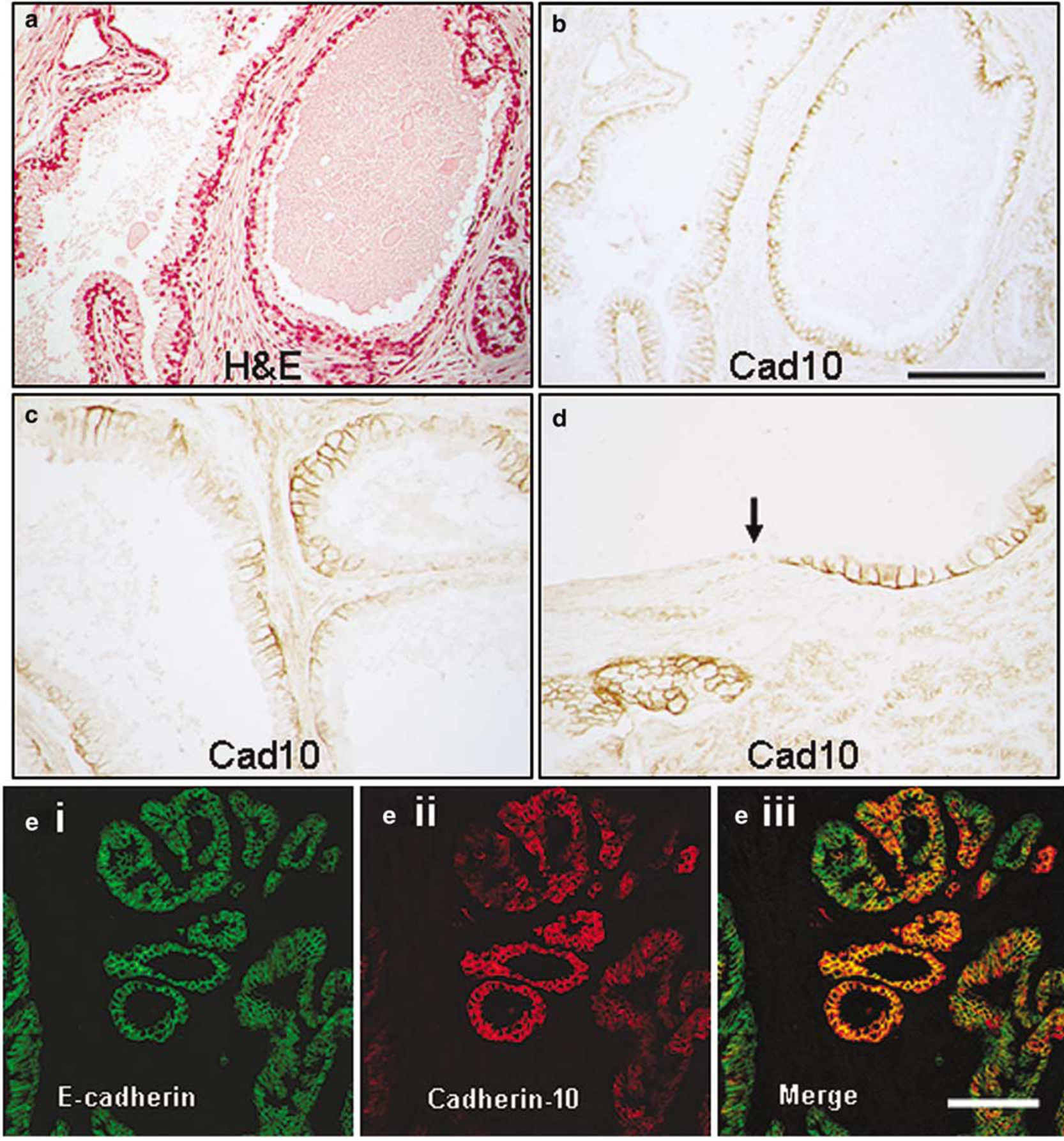

Figure 1 Cadherin-10 expression in normal prostate epithelium. (a-e) Haematoxylin and eosin (H\&E) stained section (a) with cadherin10 immunohistochemical localisation (Cad-10) in a serial section (brown deposit in b); (c and d) higher magnification micrographs of cadherin-10 immunohistochemical reaction product (brown) in prostate epithelium. (ei-eiii) Frozen sections costained for E-cadherin (ei) (green) and cadherin-10 (eii) (red). The arrow in (d) indicates a point of transition between cadherin-10-negative and -positive epithelium. Bar in B represents $150 \mu \mathrm{m}$ in (a and b), and $75 \mu \mathrm{m}$ in (c and d). Bar in eiii represents $125 \mu \mathrm{m}$ in (ei-eiii).

cadherin-10-positive luminal epithelial cells (late intermediate cells) (Figure 4c and di-iii), and was weakly expressed or absent in others (Figure 4b and di-iii). Cadherin-10 expression could change abruptly, from strongly positive to absent, in an individual gland profile where luminal cells are CK19 positive (Figure 4di-iii).
Cadherin-10 and the Luminal Cell Marker, CK18, in Normal Human Prostate Epithelium

CK18 is well-established as a marker for luminal secretory cells, ${ }^{3,5,6,27}$ and the staining pattern we observed was consistent with this (Figure 5). CK18 is present in luminal cells, but is not observed in 

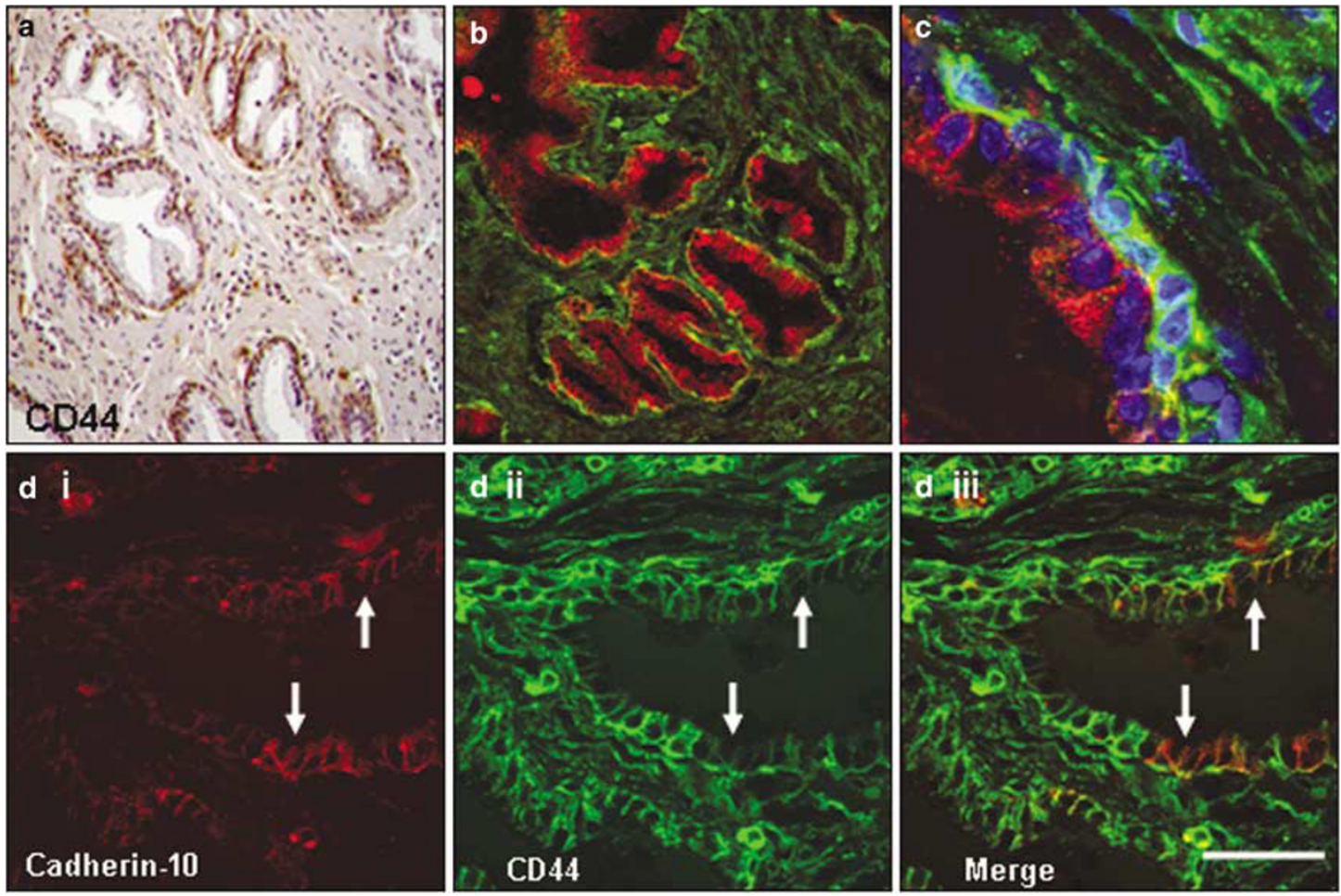

Figure 2 Cadherin-10 expression in relation to the prostate basal cell marker, CD44. (a) Immunohistochemical localisation of CD44 with haematoxylin counterstain. (b, $\mathbf{c}$ and d) Confocal microscopy after immunofluorescence costaining of CD44 (green) and cadherin-10 (red) and nucleic acid (blue in c). Arrows in (d) indicate regions of cadherin-10 high/CD44 low expression in luminal cells. Bar in diii represents $160 \mu \mathrm{m}$ in (a and b), $80 \mu \mathrm{m}$ in (c) and $40 \mu \mathrm{m}$ in (d).
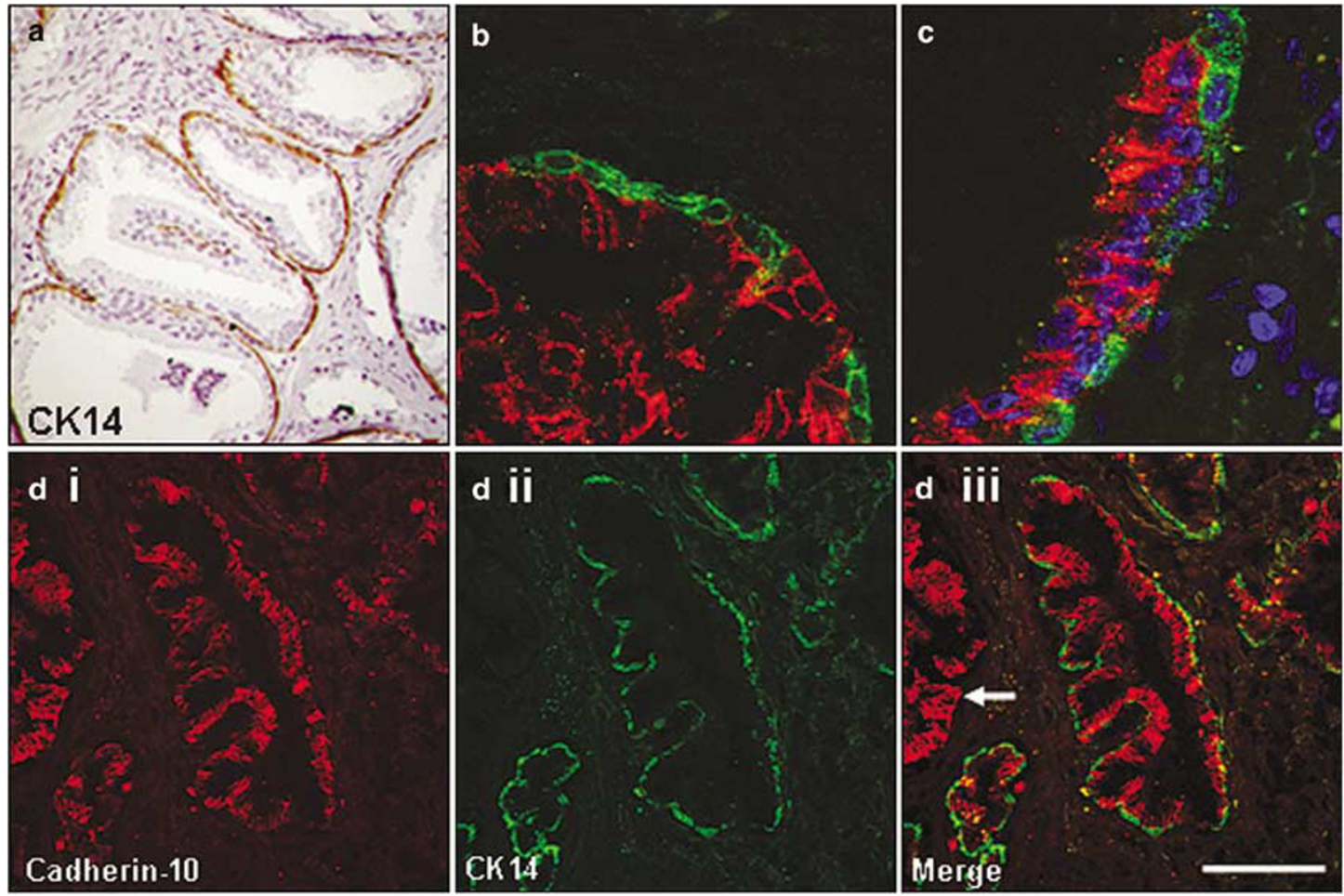

Figure 3 Costaining of cadherin-10 and the basal cell marker, cytokeratin 14 (CK14), and cadherin-10 in prostate. (a) Immunohistochemical localisation of CK14 with haematoxylin counterstain. (b, $\mathbf{c}$ and $\mathbf{d})$ Confocal microscopy after immunofluorescence costaining of CK14 (green) and cadherin-10 (red) and nucleic acid (blue in c). The arrow in (diii) indicates a cadherin-10-positive gland lacking CK14 + ve basal cells. Bar in (diii) represents $100 \mu \mathrm{m}$ in (a), $32 \mu \mathrm{m}$ in (b), $16 \mu \mathrm{m}$ in (c), $160 \mu \mathrm{m}$ in (d). 

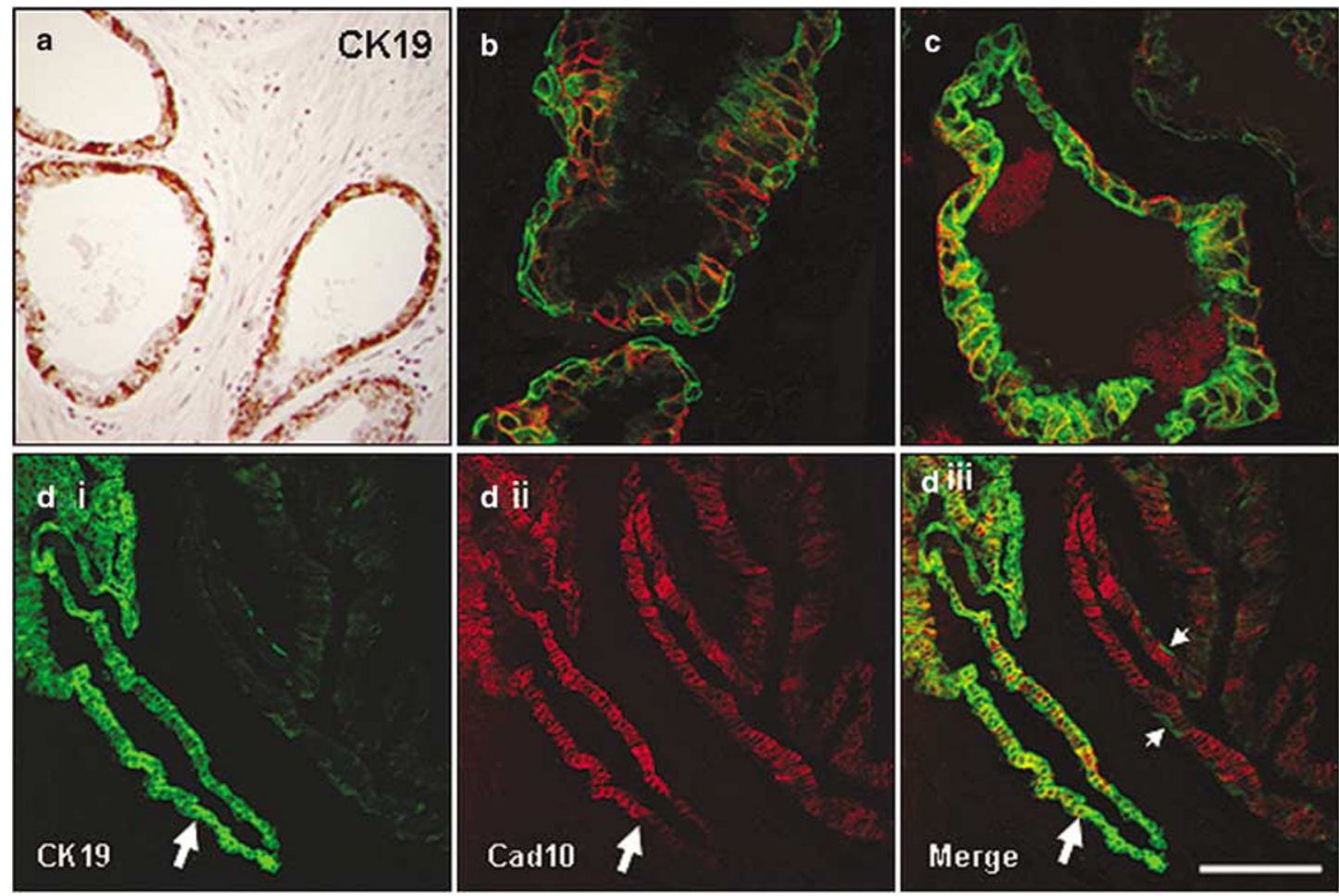

Figure 4 Costaining of the prostate intermediate cell marker, cytokeratin 19 (CK19), and cadherin-10. (a) Immunohistochemical localisation of CK19 with haematoxylin counterstain. (b, c and d) Confocal microscopy after immunofluorescence costaining of CK19 (green) and cadherin-10 (red). The arrows in (d) indicate a point of transition between cad-10-positive and -negative CK19-expressing luminal cells. Small arrows in (diii) indicate CK19 + ve basal cells. Bar represents $40 \mu \mathrm{m}$ in (b) and (c) and $160 \mu \mathrm{m}$ in (a) and (d).
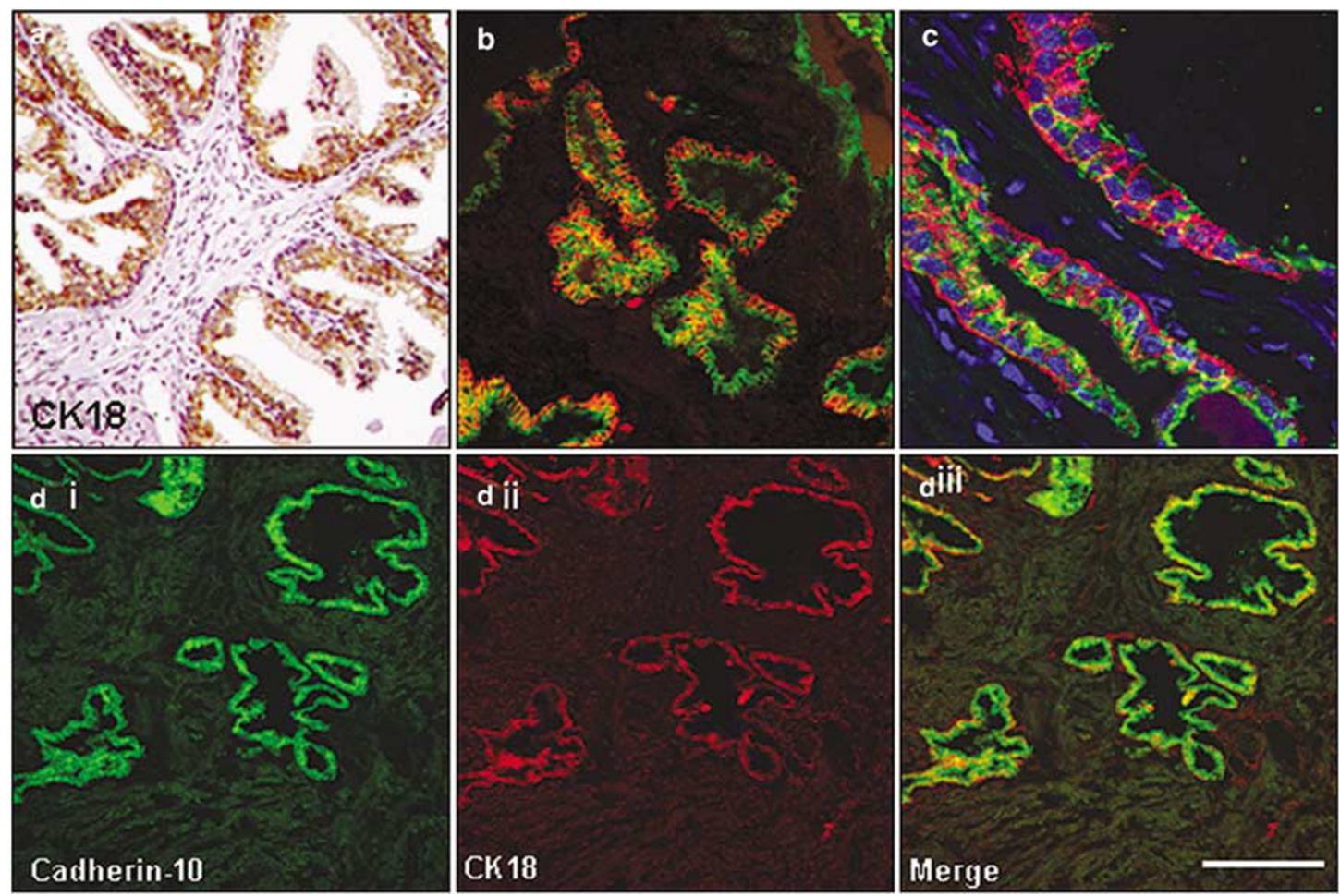

Figure 5 Costaining of the prostate luminal cell marker, cytokeratin 18 (CK18) and cadherin-10. (a) Immunohistochemical localisation of CK18 with haematoxylin counterstain. (b, $\mathbf{c}$ and $\mathbf{d}$ ) Confocal microscopy after immunofluorescence costaining of CK18 (green) and cadherin-10 (red). Bar in (diii) represents $160 \mu \mathrm{m}$ in (a), $320 \mu$ in (b) and (d), and $40 \mu \mathrm{m}$ in (c). 

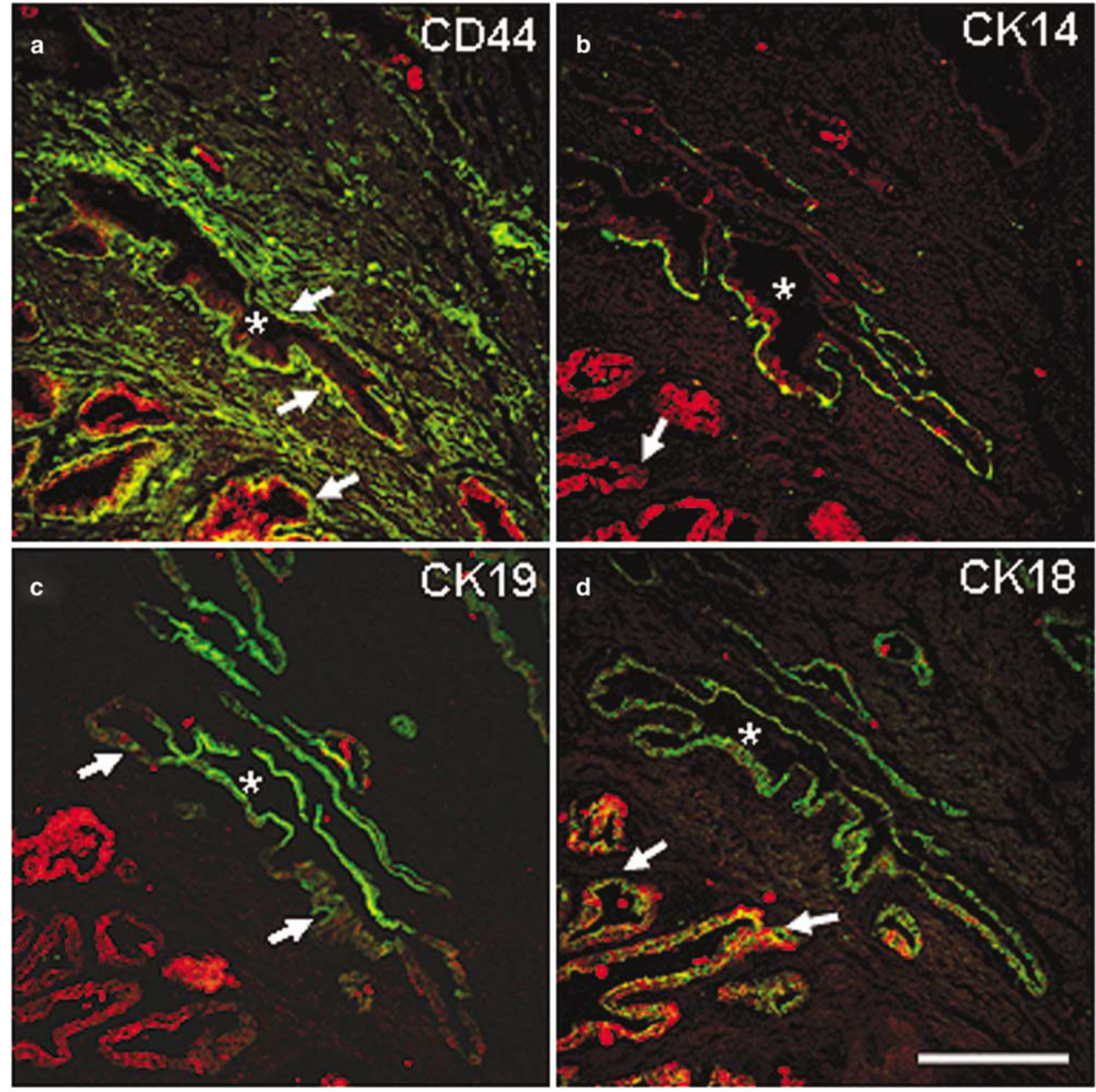

Figure 6 Cadherin-10 (red) and differentiation marker-expression (green) in serially sectioned prostate. (a) CD44 is localised to basal cells in all glands (arrows). (b) Cytokeratin 14 (CK14) is present basal cells in limited regions of the large weakly cadherin-10-positive (red) central gland profile $\left({ }^{*}\right.$ ), while absent from the CD44 + ve basal cells of strongly cadherin-10-positive glands (arrow). (c) Cytokeratin 19 (CK19) is strongly and continuously expressed in luminal cells in some regions of the central gland (*), but absent, or present in basal cells, in other regions (arrows). (d) Cytokeratin 18 (CK18) is weakly expressed in the luminal cells of the central gland $\left({ }^{*}\right)(\mathrm{CK} 19+$ ve in c) and strongly expressed in the glands (arrows) that are CK19-ve in (c). Bar represents $320 \mu \mathrm{m}$.

cells in basal positions. Cadherin-10 was consistently expressed in CK18-positive cells (Figure 5).

\section{Cadherin-10 and Differentiation Markers in Serial Sections of Normal Human Prostate Epithelium}

Prostate epithelial differentiation markers (CKs 14, 18 and 19, and CD44) were individually double- stained with cadherin-10 in serial sections of prostate. In Figure 6, two sets of morphologically normal gland profiles are evident: one set in the centre of the field (marked *), which weakly expressed cadherin-10 (cad10 $0^{ \pm}$, and a second set strongly expressing cadherin-10 $\left(\operatorname{cad} 10^{+}\right)$(Figure 6). The cad $10^{+}$profiles were associated with CD44-positive basal cells (Figure 6a), which did not express CK14 (Figure 6b), while the basal cells of 
the cad10 ${ }^{ \pm}$glands expressed both CD44 and CK14, with some CK19-positive basal cells being evident (Figure $6 \mathrm{a}, \mathrm{b}$ and $\mathrm{c}$ ). In the cad $10^{ \pm}$glands, the luminal cells coexpressed CK19 and CK18, though in some regions of adjacent epithelium, only CK18 was expressed (Figure $6 \mathrm{c}$ and $\mathrm{d}$ ). The cad $10^{+}$gland luminal cells expressed CK18, but not CK19.

\section{Cadherin-10 in Prostate Cancer}

Cadherin-10 expression was examined in prostate cancer (Figure 7). Cadherin-10 staining was low or absent in morphologically identified cancer, while in adjacent glands exhibiting prostate intraepithelial neoplasia, cadherin-10 staining was variable (Figure $7 \mathrm{a}$ and b). Costaining cadherin-10 with E-cadherin showed that in many morphologically normal glandular profiles, both cadherins were expressed, but in adjacent small, irregular cancer profiles (of Gleason Grade 4+3), E-cadherin was present, while cadherin-10 was not detected (Figure 7ci-iii). To examine the relationship between the expression of the differentiation markers (CKs 14, 18 and 19, and CD44) and cadherin-10 in prostate cancer, serial sections of a cancer patient sample, each stained for cadherin-10 and costained for one of the markers, were examined (Figure 7ei-iv). In a region of small irregular adenocarcinoma, we observed that the basal cell markers CD44 and CK14 were weakly expressed or absent (Figure 7di and ii), but the intermediate cell marker, CK19 and the luminal cell marker, CK18, were variably expressed in the cancer cells, while cadherin-10 was absent (Figure 7diii and iv). No significant cadherin-10 expression was observed at any of the Gleason grades of adenocarcinoma in the seven patient samples we analysed (Table 1).

\section{Discussion}

Our examination of the relationship between the expression of differentiation markers and cadherin10 expression in human prostate epithelium suggests that the expression of this adhesion molecule is associated with the late stages of prostate luminal secretory epithelial cell differentiation in normal glands, and that this molecule is not expressed by prostate cancer cells (Figure 8). In morphologically normal glands, we did not observe cadherin-10 in basal cells, and cadherin-10 was not coexpressed with basal cell markers. The intermediate cell marker, CK19, is expressed in cells in basal positions (early intermediate cells) and in cells in luminal positions (mid-stage $\left(\mathrm{CK} 19^{+} \mathrm{CK} 18^{-}\right)$and late intermediate cells $\left(\mathrm{CK} 19^{+} \mathrm{CK} 18^{+}\right)$). Cadherin10 was not present in CK19-positive basal cells, but was variably expressed in $\mathrm{CK}_{1} 9^{+}$cells in luminal positions. We interpreted these findings, together with the observations of differentiation markers in serial sections, as indicating that mid-stage intermediate cells $\left(\mathrm{CK} 19^{+} \mathrm{CK} 18^{-}\right.$in luminal position) were cadherin-10-low or -negative, and that as they acquired the secretory luminal cell marker, CK18, they expressed cadherin-10 (Figure 8). All CK18 expressing cells in morphologically normal glands also expressed cadherin-10. Late intermediate cells $\left(\mathrm{CK} 19^{+} \mathrm{CK} 18^{+}\right)$typically expressed cadherin-10 at lower levels than in secretory luminal cells $\left(\mathrm{CK} 19^{-} \mathrm{CK} 18^{+}\right)$. It should be noted that we observed that E-cadherin was present at all stages of differentiation in both basal and luminal cells.

The sequence of events we describe is based on a generally accepted linear progression model of differentiation of prostate epithelial cells, ${ }^{2-7}$ where basal cells are proposed to lose basal cell characteristics as they acquire luminal cell properties (Figure 8). However, Cunha and colleagues have proposed an alternative model, based on developmental evidence and the phenotype of cultured prostate epithelial cells. ${ }^{26,29}$ In this model, a small population of basal stem cells, expressing all of the common basal and luminal markers, give rise to two distinct lineages: a basal cell lineage in which the phenotype develops with the loss of luminal cell markers, and a second lineage, differentiating, via intermediate cells, towards the luminal secretory cell phenotype involving loss of basal cell markers. While this model is still somewhat controversial, the pattern of expression of cadherin-10 that we observed is compatible with either model, because it is restricted to late intermediate cells and luminal secretory cells.

The restricted expression of cadherin-10 suggests that it is part of the latter stages of the differentiation process of prostate luminal secretory cells, and that it has an important role in determining the phenotype and function of these cells. The role that this cadherin plays is not yet clear, but its association with known tissue barriers indicates that it may be involved in strengthening intercellular adhesion to reduce barrier permeability. Cadherin-10 is present in blood-brain barrier (and retinal) endothelium, but not in the endothelial cells of the more permeable non-CNS vessels. ${ }^{23}$ In addition, it is absent from the endothelium of the 'leakier' vessels of the brain circumventricular organs, and present in the adjacent ependymal neuroepithelial cells that are known to assume local barrier functions in circumventricular organs. Preliminary observations of restricted cadherin-10 expression, in relation to the more general expression of E-cadherin, in the epithelia of the gastrointestinal tracts of both human and mouse (unpublished observations) also suggest a role in the organisation of tissue barriers.

The lack of expression of cadherin-10 in prostate cancer distinguishes it from other markers of luminal cell phenotype and function. Prostate cancer cells express both basal cell and differentiated secretory cell characteristics. ${ }^{2-7}$ They typically express intermediate cell markers (eg, CK19) and secretory cell-specific components such as 

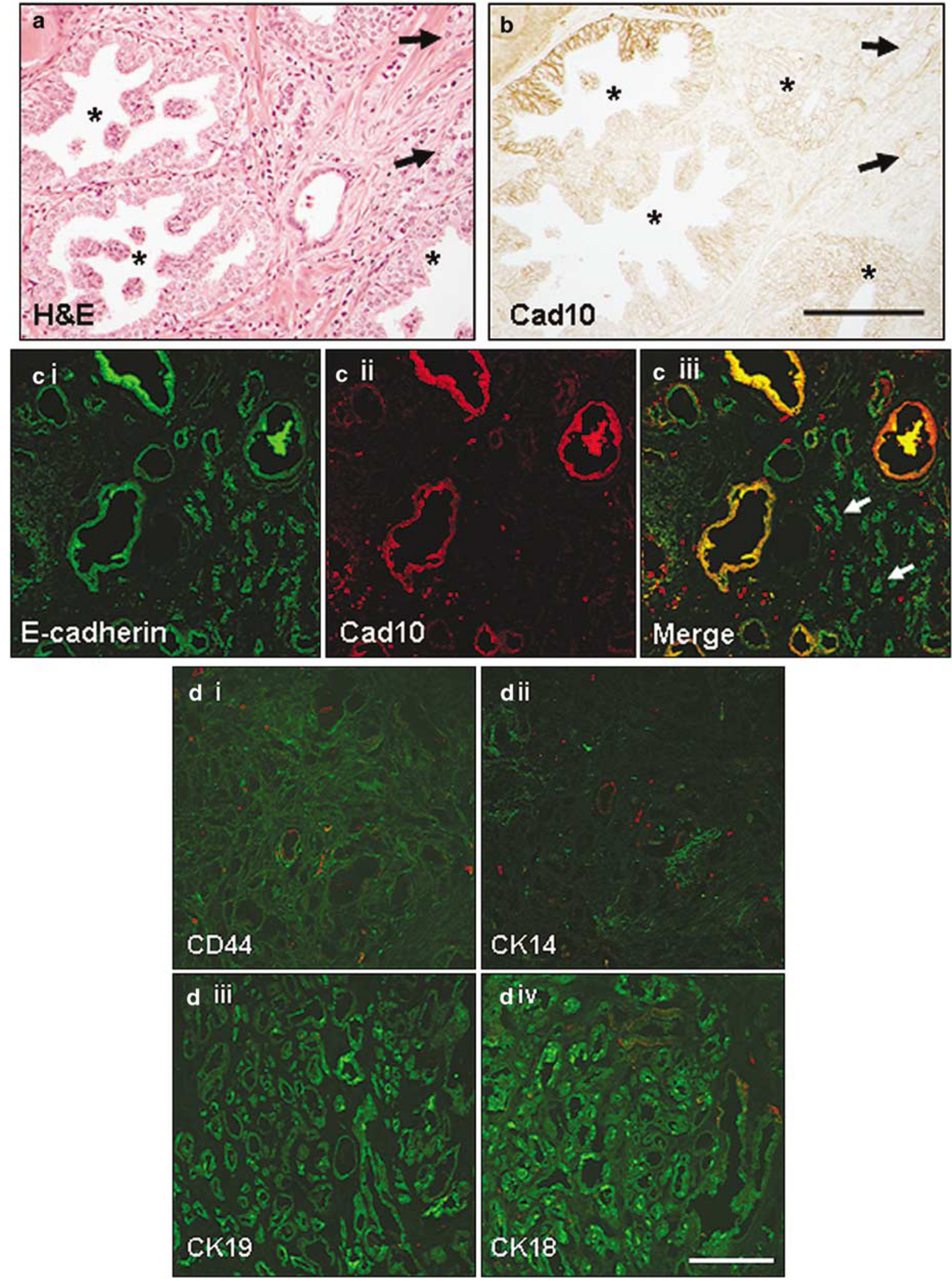

Figure 7 Cadherin-10 is absent in prostate cancer. (a) H\&E-stained prostate and (b) cadherin-10 immunohistochemistry in a section serial to (a). Arrows in (a) and (b) indicate carcinoma. Asterisks ( ${ }^{*}$ ) indicate prostate intraepithelial neoplasia. (ci-iii) Confocal costaining of a region of prostate exhibiting morphologically normal glands that are E-cadherin and cadherin-10-positive adjacent to numerous profiles of small irregular E-cadherin-positive cancerous gland that lack cadherin-10 (arrows). (di-iv) Confocal microscopy of serial sections of a region dense with cancerous gland profiles stained for cadherin-10 (red) and one of each of the differentiation markers (green). Bar in (b) represents $150 \mu \mathrm{m}$ in (a) and (b). Bar in (div) represents $400 \mu \mathrm{m}$ in (c) and (d). 
94

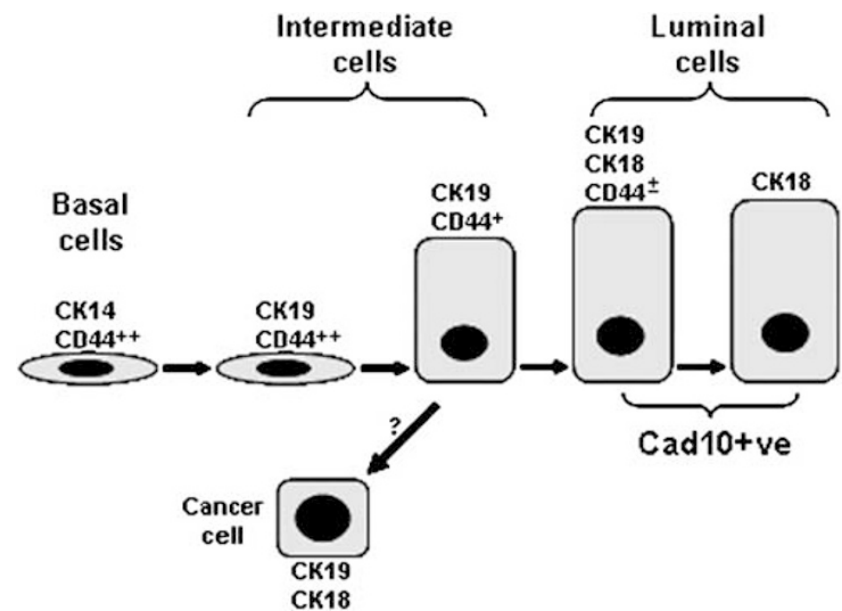

Figure 8 Scheme of the expression of cadherin-10 in relation to differentiation of prostate epithelium. A representation of the progression of differentiation and sequence of expression of the differentiation markers (CK 14, 18 and 19, CD44) used in the present study is based on previous findings. ${ }^{2-7}$ Cadherin-10 is expressed only in luminal cells at the latter stages of differentiation. Lack of cadherin-10 and basal marker expression in prostate cancer cells suggests that they arise from an intermediate cell type. E-cadherin is expressed at all stages examined.

CK8/18, androgen receptor, prostate-specific antigen and prostatic acid phosphatase. Some basal cellspecific components are absent in prostate cancer cells (eg, CK 14), while others (eg, CK5), including some involved in promoting proliferation and inhibiting apoptosis (eg, Bcl-2), are present. Accumulating evidence suggests that the bulk of prostate cancer arises from self-renewing cancer stem cells whose progeny undergo differentiation to cancer cells of low proliferative and tumour-forming capacity. ${ }^{29-32}$ The origin of these cells (ie, the cellular target of malignant transformation) is not clear, though the predominance of the intermediate/secretory phenotypes in cancer suggest an early intermediate cell target. E-cadherin ${ }^{+}$/ $\mathrm{CK}{ }^{+} / \mathrm{CK} 18^{+}$cancer cells lack cadherin-10. While cancer cells can clearly differentiate to a significant degree, the absence of cadherin-10, even at low Gleason scores, is consistent with a failure to reach a fully differentiated state.

Our findings suggest that cadherin-10 plays a key role in prostate epithelial differentiation, and thus prostate disease. E-cadherin is expressed at all stages of differentiation in normal prostate epithelium, and is present in prostate cancer, except at the highest Gleason scores. ${ }^{33-35}$ The restriction of cadherin-10 expression to secretory luminal cells and its absence from cancer cells indicate a key role in normal tissue organisation. The lack of cadherin-10 in cancer cells might contribute to the altered morphology and behaviour of these cells. It will be important to determine the mechanisms controlling the expression of cadherin-10, and to elucidate the cell biology of cadherin-10 in epithelial cells. In doing so, it is likely that important differences between benign and malignant prostate epithelium will be revealed.

\section{Acknowledgement}

This work was supported by the Wellcome Trust. We thank Lorraine Lawrence for excellent technical assistance. MJA was supported by a Monroe Scholar Research Scholarship, College of William and Mary, Williamsburg, Virginia 23185, USA.

\section{Conflict of interest}

The authors report no conflict of interest pertaining to this research.

\section{References}

1 McNeal JE. Normal histology of the prostate. Am J Surg Pathol 1988;12:619-633.

2 Bonkhoff H, Stein U, Remberger K. The proliferative function of basal cells in the normal and hyperplastic human prostate. Prostate 1994;24:114-118.

3 Hudson DL. Epithelial stem cells in human prostate growth and disease. Prostate Cancer Prostatic Dis 2004;7:188-194.

4 Isaacs JT, Coffey DS. Etiology and disease process of benign prostatic hyperplasia. The Prostate 1989; 2:33-50.

5 Long RM, Morrissey C, Fitzpatrick JM, et al. Prostate epithelial cell differentiation and its relevance to the understanding of prostate cancer therapies. Clin Sci (London) 2005;108:1-11.

6 van Leenders GJ, Schalken JA. Epithelial cell differentiation in the human prostate epithelium: implications for the pathogenesis and therapy of prostate cancer. Crit Rev Oncol Hematol 2003;46(Suppl): S3-S10.

7 Robinson EJ, Neal DE, Collins AT. Basal cells are progenitors of luminal cells in primary cultures of differentiating human prostatic epithelium. Prostate 1998;37:149-160.

8 Gumbiner BM. Regulation of cadherin-mediated adhesion in morphogenesis. Nat Rev Mol Cell Biol 2005;6:622-634.

9 Takeichi M, Nakagawa S, Aono S, et al. Patterning of cell assemblies regulated by adhesion receptors of the cadherin superfamily. Philos Trans R Soc Lond 2000;355:885-890.

10 Conacci-Sorrell M, Zhurinsky J, Ben-Ze'ev A. The cadherin-catenin adhesion system in signaling and cancer. J Clin Invest 2002;109:987-991.

11 Hajra KM, Fearon ER. Cadherin and catenin alterations in human cancer. Genes Chromosomes Cancer 2002;34:255-268.

12 Van Aken E, De Wever O, Correia da Rocha AS, et al. Defective E-cadherin/catenin complexes in human cancer. Virchows Arch 2001;439:725-751.

13 Wijnhoven BP, Dinjens WN, Pignatelli M. E-cadherincatenin cell-cell adhesion complex and human cancer. Br J Surg 2000;87:992-1005.

14 Jaggi M, Johansson SL, Baker JJ, et al. Aberrant expression of E-cadherin and beta-catenin in human prostate cancer. Urol Oncol 2005;23:402-406.

15 Morton Jr RA, Ewing CM, Watkins JJ, et al. The E-cadherin cell-cell adhesion pathway in urologic malignancies. World J Urol 1995;13:364-368. 
16 Morton RA, Ewing CM, Nagafuchi A, et al. Reduction of E-cadherin levels and deletion of the alpha-catenin gene in human prostate cancer cells. Cancer Res 1993;53:3585-3590.

17 Giroldi LA, Schalken JA. Decreased expression of the intercellular adhesion molecule E-cadherin in prostate cancer: biological significance and clinical implications. Cancer Metastasis Rev 1993;12:29-37.

18 Umbas R, Schalken JA, Aalders TW, et al. Expression of the cellular adhesion molecule E-cadherin is reduced or absent in high-grade prostate cancer. Cancer Res 1992;52:5104-5109.

19 Macoska JA. Ancestry, genetic susceptibility, E-cadherin-160A and prostate cancer risk-is there an association? J Urol 2006;176:435-436.

20 Alexander NR, Tran NL, Rekapally H, et al. N-cadherin gene expression in prostate carcinoma is modulated by integrin-dependent nuclear translocation of Twist1. Cancer Res 2006;66:3365-3369.

21 Jaggi M, Nazemi T, Abrahams NA, et al. N-cadherin switching occurs in high Gleason grade prostate cancer. Prostate 2006;66:193-199.

22 Mariotti A, Perotti A, Sessa C, et al. N-cadherin as a therapeutic target in cancer. Expert Opin Investig Drugs 2007;16:451-465.

23 Williams MJ, Lowrie MB, Bennett JP, et al. Cadherin-10 is a novel blood-brain barrier adhesion molecule in human and mouse. Brain Res 2005;1058:62-72.

24 Kools P, Vanhalst K, Van den Eynde E, et al. The human cadherin-10 gene: complete coding sequence, predominant expression in the brain, and mapping on chromosome 5p13-14. FEBS Lett 1999;452:328-334.

25 Alam TN, O’Hare MJ, Laczko I, et al. Differential expression of CD44 during human prostate epithelial cell differentiation. J Histochem Cytochem 2004;52: 1083-1090.

26 Wang Y, Hayward S, Cao M, et al. Cell differentiation lineage in the prostate. Differentiation 2001;68:270-279.

27 Yang Y, Hao J, Liu X, et al. Differential expression of cytokeratin mRNA and protein in normal prostate, prostatic intraepithelial neoplasia, and invasive carcinoma. Am J Pathol 1997;150:693-704.

28 Peehl DM, Sellers RG, McNeal JE. Keratin 19 in the adult human prostate: tissue and cell culture studies. Cell Tissue Res 1996;285:171-176.

29 Tokar EJ, Ancrile BB, Cunha GR, et al. Stem/progenitor and intermediate cell types and the origin of human prostate cancer. Differentiation 2005;73:463-473.

30 Lam JS, Reiter RE. Stem cells in prostate and prostate cancer development. Urol Oncol 2006;24:131-140.

31 Nikitin AY, Matoso A, Roy-Burman P. Prostate stem cells and cancer. Histol Histopathol 2007;22:1043-1049.

32 Tang DG, Patrawala L, Calhoun T, et al. Prostate cancer stem/progenitor cells: identification, characterization, and implications. Mol Carcinog 2007;46:1-14.

33 Koksal IT, Ozcan F, Kilicaslan I, et al. Expression of E-cadherin in prostate cancer in formalin-fixed, paraffin-embedded tissues: correlation with pathological features. Pathology 2002;34:233-238.

34 Ray ME, Mehra R, Sandler HM, et al. E-cadherin protein expression predicts prostate cancer salvage radiotherapy outcomes. J Urol 2006;176:1409-1414; discussion 14.

35 Algaba F, Arce Y, Fernandez S, et al. Adhesion molecules expression as a potential marker of prostate cancer aggressivity. A TMA study of radical prostatectomy specimens. Arch Ital Urol Androl 2006;78: 130-134. 